DOI: $10.4274 /$ gulhane.galenos.2020.1176

Gulhane Med J 2021;63:75-77

\title{
Synchronous multicentric astrocytoma with different histological types in both supratentorial and infratentorial regions
}

\author{
(D) Adem Doğan ${ }^{1}$, (1) Murat Kutlay ${ }^{1}$, (1) Mükerrem Safalı2, (1) Sait Kayhan ${ }^{1}$ \\ 1 University of Health Sciences Turkey, Gülhane Faculty of Medicine, Department of Neurosurgery, Ankara, Turkey \\ 2University of Health Sciences Turkey, Gülhane Faculty of Medicine, Department of Pathology, Ankara, Turkey
}

Date submitted:

27.05.2020

Date accepted:

15.06.2020

Online publication date:

15.03.2021

Corresponding Author:

Adem Doğan MD, University of Health

Sciences Turkey, Gülhane Faculty of

Medicine, Department of Neurosurgery,

Ankara, Turkey

drademdogan@yahoo.com

ORCID:

orcid.org/0000-0002-2881-4191

Keywords: Multicentric glioma, pilocytic astrocytoma, anaplastic astrocytoma

\begin{abstract}
Synchronous multicentric gliomas are lesions with different histological features and they occur in the same time at different parts of the brain without anatomical continuity. We aimed to describe a multicentric astrocytoma with different histopathological types located in supratentorial and infratentorial regions of the brain. Our patient was operated first for the posterior fossa tumor, then he underwent the removal of frontal tumor. The posterior fossa tumor was pilocytic astrocytoma and the frontal tumor was anaplastic astrocytoma. Synchronous multicentric astrocytoma with different histology is very rare and should be treated in different sessions for better clinical outcome.
\end{abstract}

\section{Introduction}

Gliomas are the most common malignant intracranial tumors that may be observed in any part of central nervous system including cranial and spinal regions, as well as in the spinal cord (1-3). They may be solitary or multicentric in the cranium. Multicentric gliomas are very rare and unique lesions for neurosurgeons with an incidence around 2-10\% $(1,4)$. Gliomas are defined as multicentric when no macroscopic or microscopic continuity is found between the lesions $(1,4,5)$.

Multicentric gliomas are more frequent in middle-aged men and they show rapid progression $(5,6)$. The prognosis is poor and the overall survival of these patients is usually short. These tumors are mostly seen in the supratentorial region $(1,6)$. It is very rare to observe in supra- and infratentorial locations simultaneously $(5,7)$.
We presented a unique case of multicentric glioma, which was located in both supra- and infratentorial regions with different histological types. We reported clinical, radiological and surgical features of this rare case.

\section{Case Presentation}

A 26-year-old male patient was admitted to our outpatient clinic for headache. Neurological examination was normal. The cranial magnetic resonance imaging (MRI) revealed a lesion with cystic and solid components of $38 \times 52 \times 43 \mathrm{~mm}$ in the infratentorial region. It was hypointense in T1W series, heterogeneous hyperintense in $\mathrm{T} 2 \mathrm{~W}$ series with heterogeneous contrast enhancement of solid component, presumed as pilocytic astrocytoma (Figures 1A, 1B). In addition, there was another lesion in the right frontal lobe, in the subcortical white 
matter, which was $42 \times 47 \times 41 \mathrm{~mm}$ in size, hypointense in T1W and hyperintense in T2W series (Figures 1C, 1D).

The patient was hospitalized, and 2-staged surgery was planned, first for posterior fossa tumor and the second for frontal lobe tumor. In the first stage, suboccipital craniectomy was performed and cystic white-gray tumor was totally removed. Histological diagnosis was pilocytic astrocytoma grade 1. Histologically, the tumor was composed of spindle cells (Figure 2A) showing compact development pattern in most areas on the fibrillary background and in some areas, abundant Rosenthal fibers (Figure 2B) were observed. p53 expression was negative, GFAP was strongly positive. Partially focal infiltrative pattern was observed in most areas. Ki-67 proliferation index was around $1 \%$.

In the second stage, 1-month after the first stage, right frontal tumor was removed using frontal craniotomy. Histopathological diagnosis was Anaplastic Astrocytoma grade 3. Histologically, p53 expression was widely positive, and neoplastic cells were negative for ATRX expression (Figure 2C). Ki-67 proliferation index was 10\% (Figure 2D).

The patient was discharged without neurological deficit. Chemotherapy (temozolomide $100 \mathrm{mg}$ ) and radiotherapy (60 $\mathrm{Cy} / 30 \mathrm{Fr}$ for frontal lesion) were administered after surgical treatment. The patient's follow-up at the end of the second year showed no signs of recurrence or residual in cranial MRI (Figures 1E,H). The patient's neurological condition was still stable.

\section{Discussion}

Multicentric gliomas were first reported by Bradley in 1880 (7). The frequency and etiology of multicentric gliomas are not well known $(1,6-8)$. It is important to differentiate multifocal and multicentric gliomas. Multifocal tumors are known to be caused

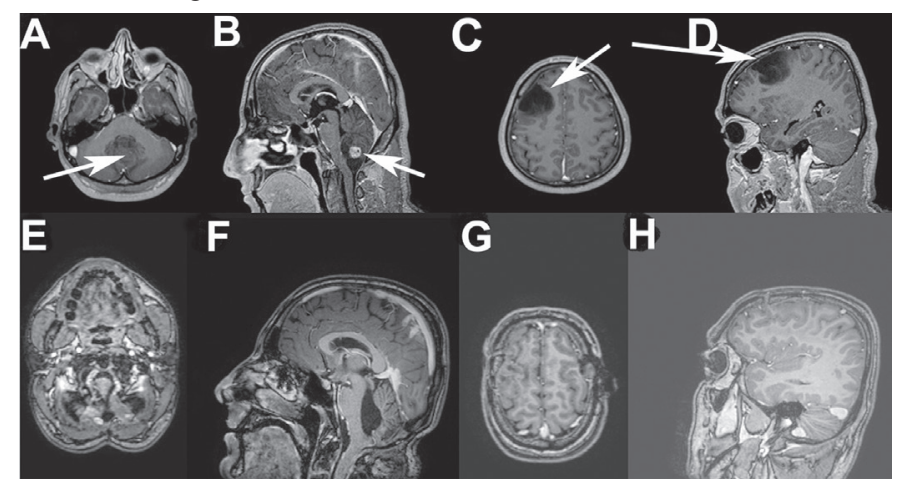

Figure 1. Preoperative axial $(A)$ and sagittal $(B) T 1 W$ magnetic resonance imaging scans show contrast-enhancing mass lesion (marked with white arrows) in the posterior fossa, axial (C) and sagittal (D) T1W images show right frontal hypointense tumor (marked with white arrows) in the supratentorial region. Postoperative axial T1W (E) and sagittal T1W (F) scans confirmed total resection of posterior fossa tumor (pilocytic astrocytoma), axial $(\mathrm{G})$ and sagittal $(\mathrm{H})$ supratentorial slices confirmed total removal of right frontal tumor (anaplastic astrocytoma) by the invasion of commissural or tract fibers, but multicentric gliomas occur in a distant region and there is no connection path between these tumors $(1,3,6-8)$.

The pathogenesis of multiple gliomas is still unclear. Willis (9) stated that this was a two-stage process. First, almost all of the brain tissue undergoes a neoplastic transformation and becomes susceptible to neoplastic development. In the next stage, abnormal cell proliferation causes a number of warnings (9). Another hypothesis was interpreted by the long-distance invasion and migration capacity of glioma cells (10).

Cerebral metastasis, brain abscesses, and lymphomas should be considered in the differential diagnosis of multicentric gliomas $(1,11)$. The exact diagnosis is made after surgical removal of tumors via classical craniotomy with microsurgical approach. However, endoscopic endonasal approach may be used for gliomas located in the skull base. Meanwhile, the reconstruction of skull base defect is always a challenge for endoscopic approach (12). In our patient, the diagnosis of supratentorial tumor was anaplastic (grade 3) astrocytoma and the infratentorial tumor was a pilocytic (grade 1) astrocytoma. The time period between the 2 surgeries was one month.

Inoue et al. (5) reported a case of multicentric glioma similar to our case, but not presented at the same time. In their case, infratentorial tumor was detected 7 months after the surgery for supratentorial glioma. In addition, the diagnosis of supratentorial tumor was diffuse (grade 2) astrocytoma and infratentorial tumor was glioblastoma (grade 4). In our case, the diagnosis of supratentorial was anaplastic astrocytoma (grade 3) and infratentorial was pilocytic astrocytoma (grade 1). As mentioned above, multicentric glioma cases are mostly seen

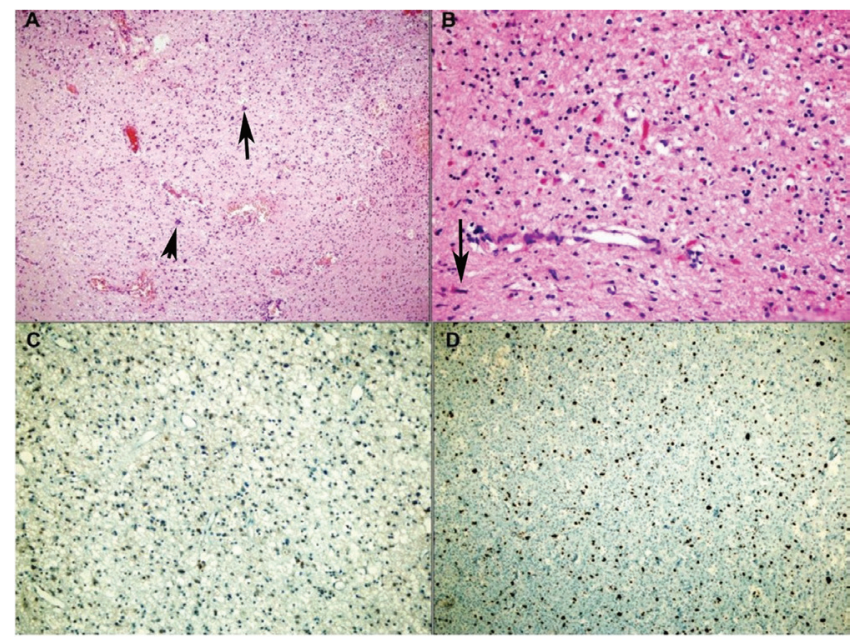

Figure 2. A) Histological examination shows that the tumor was composed of spindle cells (marked with black arrows). B) There is compact development pattern in most areas on the fibrillary background and in some areas abundant Rosenthal fibers (marked with black arrow). C) Immunohistochemical examination shows that p53 expression was widely positive, negative for ATRX neoplastic cells. D) Ki-67 proliferation index was $10 \%$ 
in the supratentorial region $(1,4,6,7)$. Nakhl et al. (6) presented a case of multicentric glioma but all tumors were located in supratentorial region. In our case, the tumors were in different regions and there was no connection between the tumors.

Predisposing factors for multicentric gliomas are neurofibromatosis type 1 , multiple sclerosis, and radiation exposure $(1,2,7)$. Especially, radiation is an important factor on the development of brain gliomas $(2,13)$. Multicentricity is usually associated with poor prognosis $(1,10)$. Our case was a young male patient. He had no radiation history, no other systemic disease such as neurofibromatosis and genetic disorders. He underwent successful removal of both tumors. There was no recurrence in the second year follow-up with adjuvant chemotherapy and radiotherapy in the postoperative period.

\section{Conclusion}

Synchronous multicentric astrocytomas in two different regions of the brain with different histological diagnosis are very rare. These tumors should be removed in different sessions with a reasonable time period. In addition to microscopic spread, genetic factors may also have an effect on pathophysiology of multicentric astrocytomas. Proper surgical intervention and postoperative adjuvant treatments have significantly contributed to the patient's survival.

\section{Ethics}

Informed Consent: The patient gave written informed consent to publish the case report without revealing his identity.

Peer-review: Externally peer-reviewed.

\section{Authorship Contributions}

Surgical and Medical Practices: M.K., Design: A.D., Data Collection or Processing: M.S., Analysis or Interpretation: M.S., Literature Search: A.D., Writing: A.D., S.K.

Conflict of Interest: No conflict of interest was declared by the authors.

Financial Disclosure: The authors declared that this study received no financial support.

\section{References}

1. İzci Y, Gürkanlar D, Timurkaynak E. Multicentric gliomas: Still remains a controversial issue. Report of three cases and literature review. Turk Neurosurg. 2005;15:71-75.

2. Doğan A, Yasar S, Kayhan S, Kırmızıgöz Ş, Kaplan A. Brain and space. Türk Nöroşir Derg. 2018;28:372-376.

3. Durmaz MO, Doğan A, Ezgü MC, Özer Mi, Yaşar S, Kırık A. Primary spinal cord glioblastoma in young population: Report of two cases. J Turk Spinal Surg. 2020;31:55-59.

4. Barnard RO, Geddes JF. The incidence of multifocal cerebral gliomas. A histologic study of large hemisphere sections. Cancer. 1987;60:1519-1531.

5. Inoue A, Ohnishi T, Kohno S, et al. A case of multicentric gliomas in both supra- and infratentorial regions with different histology: a case report. World J Surg Oncol. 2016;14:152.

6. Nakhl F, Chang EM, Shiau JSC, et al. A patient with multiple synchronous gliomas of distinctly different grades and correlative radiographic findings. Surg Neurol Int. 2010;1:48.

7. Salvati M, Caroli E, Orlando ER, Frati A, Artizzu S, Ferrante L. Multicentric glioma: our experience in 25 patients and critical review of the literature. Neurosurg Rev. 2003;26:275279.

8. Kotwica Z, Papierz W. Cerebral and cerebellar glial tumors in the same individual. Neurosurgery. 1992;30:439-441.

9. Willis RA. Pathology of tumors. London: Butterworth; 1960:811.

10. Patil CG, Eboli P, Hu J. Management of multifocal and multicentric gliomas. Neurosurg Clin N Am. 2012;23:343350.

11. Kırık A, Cansever T, Erdogan E. Surgical treatment of brain metastasis. Türk Nöroşir Derg.2008;18:162-172.

12. Kutlay M, Durmaz O, Kırık A, et al. Sellar defect reconstruction with vascularized superior turbinate mucosal flaps in endonasal endoscopic transsellar approach. World Neurosurg. 2020;133:503-512.

13. Izci Y. Precision medicine in brain gliomas. In: Precision medicine in cancers and non-communicable diseases. Boca Raton, FL; CRC Press, Taylor \& Francis Group; 2018:39-48. 